\title{
Reliability analysis of two-unit cold standby repairable systems under Poisson shocks
}

\author{
Qingtai $\mathrm{Wu}^{1}$, Shaomin $\mathrm{Wu}^{2}$
}

\begin{abstract}
This paper analyses the reliability of a cold standby system consisting of two repairable units, a switch and a repairman. At any time, one of the two units is operating while the other is on cold standby. The repairman may not always at the job site, or take vacation. We assume that shocks can attack the operating unit. The arrival times of the shocks follow a homogeneous Poisson process and their magnitude is a random variable following a known distribution. Time on repairing a failed unit and the length of repairman's vacation follow general continuous probability distributions, respectively. The paper derives a number of reliability indices: system reliability, mean time to first failure, steady-state availability, and steady-state failure frequency.
\end{abstract}

Key words: Poisson shock, cold standby system, vector Markov process, reliability indices.

\section{Introduction}

The shock model, one of the important models in the reliability theory, has been extensively studied in the last decades and results are summarized in [1]. The main interest of existing research 1 College of Science, Nanjing Agricultural University, Nanjing, 210095, China. Corresponding author.Email:wuqingtai@njau.edu.cn.

2 School of Applied Sciences, Cranfield University, Cranfield, Bedfordshire MK43 0AL, United Kingdom 
focuses on one-unit systems with Poisson shocks under the assumption that the damage to the system resulted from a single shock can be neglected, and the system fails once the damage has accumulated to a certain level. For example, Shamthikumar and Sumita studied the earthquake and inventory problems and introduced a shock model, in which the system fails when the magnitude of a shock exceeds a pre-specified threshold [2,3]. Li et al. studied complex systems consisting of $n$ i.i.d. units with a $\delta$-shock model [4].

Standby systems have attracted the attention of many researchers. There are three main types of redundant standby systems: cold, warm and hot. Cold standby systems have been studied extensively in the past. For example, $[5,6]$ have investigated two-unit standby system models and assumed that the standby unit is immediately switched on once the operating unit fails, whereas Gupta and Kishan [7] assume that the standby unit is not immediately switched before a fixed preparation time is took to put standby into service. Recently, the reliability indices of cold standby repairable systems have been derived when the times between repairs are assumed to follow the geometric process $[8,9]$. Meanwhile, Mahmoud and Moshrefa [10] deal with the study of the stochastic analysis of a two-unit cold standby system considering hardware failure, human error failure and preventive maintenance.

In the research mentioned above, a unit is repaired immediately after it fails, which might not be the case in practice: in most real scenarios, a failed unit might not be repaired immediately due to various reasons. One of the problems often occurred, for example, is the absence of maintenance staff. This happens in median or small firms as they might not be able to afford to recruit a full-time repairman looking after their equipment. Instead, a repairman might need to care many types of equipments and he might not be able to repair a failed unit immediately once it fails. We say that the repairman is on vacation if he is absent when an unit fails, although he might actually be repairing other equipment.

Doshi [11] provides a comprehensive survey on vacation system models, and Ke and Wang [12] investigated a machine repair problem with two vacation policies (single vacation and multiple 
vacations), both of which were based on a queuing theory viewpoint. Su and Shi [13] discussed the reliability of a $n$-unit series system in which the repairman takes multiple vacations. Jia and $\mathrm{Wu}[14]$ studied a replacement policy for a repairable system with its repairman taking multiple vacations. Jia and $\mathrm{Wu}[15]$ develop replacement policy for a cold standby system composed of two identical units with perfect switching.

The reliability indices of a two-unit cold standby repairable systems are important to industries. Analysing and deriving such indices for the systems impacted by shocks can be more interesting as shocks occur from time to time in the real world. This motivates us to analyse the reliability of a two-unit cold standby repairable system. We assume the system might be attacked by shocks following a Poisson process. It should be noted such analysis is not an easy task when the survivor distribution and the distribution of the vacation period of the repairman are general distributions.

In this paper, we introduce a supplementary variable when solving the partial differential equations used to describe the dynamics between state transitions. With the help of the ergodicity of the investigated process and the theory of the first-order, linear, ordinary differential equations, we obtain explicit expressions of reliability indices such as steady-state availabilities and steady state failure frequency.

The paper is structured as follows. Section 2 describes the system and lists assumptions. Section 3 derives integro-differential equations. In section 4, we transform the integro-differential equations into the first-order, linear, ordinary differential equations, and obtain the explicit solution of the equations. Explicit expressions for reliability indices for the system are derived. Section 5 presents a special case (model) without vacation. Section 6 offers numerical examples. Concluding remarks are offered in the last section. 


\section{Model assumptions}

Assume that the system under discussion is a cold standby repairable system. A part-time repairman looks after the system that might be attacked by shocks. The following assumptions hold.

A1. The system consists of two different units (ie, unit 1 and unit 2), a switch and a repairman. The two units are operating alternatively: one unit is operating while the other is on cold standby or is being repaired if it has failed. The standby unit will be switched to the operating state once the operating unit fails, the switch is perfect.

A2. The system subjects to shocks. The arrivals of the shocks follow a Poisson process $\{N(t), t \geq 0\}$ with the intensity $\lambda>0$. The magnitude of each shock, $\widehat{X}$, is an independent random variable with distribution function $F$.

A3. When a shock arrives, it only affects the operating unit. The operating unit will fail when the magnitude of a shock exceeds a threshold. The threshold of unit $i$ is a non-negative random variable $\tau_{i}$ with a distribution function $\Phi_{i},(i=1,2)$.

A4. When a unit fails with the presence of the repairman, it will be repaired immediately. Once the failed unit is repaired, the repairman leaves for a time period (maybe for other tasks), or is said to take a vacation. The repair rule is "first-in-first-out". If a unit fails when the other is being repaired, the newly failed unit must wait for repair and the system is down. If two units are waiting for repair when the repairman returns from a vacation, unit 1 has the priority to be repaired ${ }^{3}$. If there are no failed unit when the repairman returns from a vacation, he does not take a vacation again and remains idle until the first failed unit appears.

Denote $Y_{i}(\mathrm{i}=1,2)$ as unit $i$ 's repair time, and $Z$ as the vacation length of the repairman denoted. Their distributions are: $H_{i}(t)=\int_{0}^{t} h_{i}(x) \mathrm{d} x=1-e^{-\int_{0}^{t} \mu_{i}(x) \mathrm{d} x}, V(t)=\int_{0}^{t} v(x) \mathrm{d} x=$

$\overline{3}$ This is a realistic assumption as the repairman, upon his return from vacation, might pick up one of the failed units to repair and it is not important for him to select which unit — it can be either unit 1 or unit 2 . 
$1-e^{-\int_{0}^{t} \alpha(x) \mathrm{d} x}$, respectively. We also denote $E(Z)=\frac{1}{\alpha}, \bar{V}(t)=1-V(t), E\left(Y_{i}\right)=\frac{1}{\mu_{i}}, \overline{H_{i}}(t)=$ $1-H_{i}(t)(i=1,2)$.

A5. Shocks are assumed to be the only cause of unit failure, and the system fails only if both the units fail.

A6. All random variables are independent. At the beginning, the two units are new, unit 1 starts to work, unit 2 is on cold standby, and the repairman takes vacation. The units can be repaired "as good as new".

\section{Model development}

With the model assumptions given in the preceding section, the failure probability of unit $i$, given the shock value $\widehat{x}$, is $\Phi_{i}(\widehat{x})=P\left(\tau_{i} \leq \widehat{x}\right)$. Since the magnitude of a shock is a random variable $\widehat{X}$, the conditional failure probability of unit $i$ is a random variable $\Phi_{i}(\widehat{X})$ with $i=1,2$, respectively, and their probability distribution can be written by: $P_{i}(x)=P\left(\Phi_{i}(\widehat{X}) \leq x\right)=P\left(\widehat{X} \leq \Phi_{i}^{-1}(x)\right)=$ $F\left(\Phi_{i}^{-1}(x)\right), 0 \leq x \leq 1,(i=1,2)$.From assumptions $\mathbf{A} \mathbf{2}$ and $\mathbf{A} \mathbf{3}$, we can see that, the probability that one shock causes unit $i$ to fail is:

$$
r_{i}=P\left(\widehat{X}>\tau_{i}\right)=\int_{0}^{\infty} P\left(\tau_{i}<\widehat{x} \mid \widehat{X}=\widehat{x}\right) \mathrm{d} P(\widehat{X} \leq \widehat{x})=\int_{0}^{\infty} \Phi_{i}(\widehat{x}) \mathrm{d} F(\widehat{x}),(i=1,2) .
$$

Let $S(t)$ be the system state at time $t$, then

state 0: at time $t$, unit 1 is operating, unit 2 is on cold standby, and the repairman is taking a vacation.

state 1: at time $t$, unit 2 is operating, unit 1 is on cold standby, and the repairman is taking a vacation.

state 2: at time $t$, unit 2 is operating, unit 1 is waiting for repair, and the repairman is taking a vacation.

state 3: at time $t$, unit 1 is operating, unit 2 is waiting for repair, and the repairman is taking 
a vacation.

state 4: at time $t$, two units are waiting for repair, and the repairman is taking a vacation. state 5: at time $t$, unit 1 is operating, unit 2 is on cold standby, and the repairman is idle. state 6: at time $t$, unit 2 is operating, unit 1 is on cold standby, and the repairman is idle. state 7: at time $t$, unit 2 is operating, unit 1 is being repaired.

state 8: at time $t$, unit 1 is operating, unit 2 is being repaired.

state 9: at time $t$, unit 1 is being repaired, unit 2 is waiting for being repaired. state 10: at time $t$, unit 2 is being repaired, unit 1 is waiting for being repaired.

The state space is $\Omega=\{0,1,2,3,4,5,6,7,8,9,10\}$, where the operating state set is $W=\{0,1,2,3,5$, $6,7,8\}$ and the failure state set is $F=\{4,9,10\}$. As the repair time has a general continuous distribution, $\{S(t), t \geq 0\}$ is not a Markov process. Therefore we introduce the following supplementary variables:

- $X(t)$ : if $S(t)=0,1,2,3,4$. Then $X(t)$ is the elapsed vacation time when the repairman is taking a vacation at time $t$.

- $Y_{1}(t)$ : if $S(t)=7,9$. Then $Y_{1}(t)$ is the elapsed repair time of unit 1 being repaired at time $t$. - $Y_{2}(t)$ : if $S(t)=8,10$. Then $Y_{2}(t)$ is the elapsed repair time of unit 2 being repaired at time $t$.

Then $\left\{\left(S(t), X(t), Y_{1}(t), Y_{2}(t)\right), t \geq 0\right\}$ is a continuous vector Markov process (see [16]) with the following state space: $\Omega^{*}=\{[0, x],[1, x],[2, x],[3, x],[4, x], 5,6,[7, y],[8, z],[9, y],[10, z]\}$. where $x, y$ and $z$ are the realisation values of $X(t), Y_{1}(t)$ and $Y_{2}(t)$, respectively. Denote:

$$
\begin{gathered}
Q_{i}(t, x)=P(S(t)=i, X(t) \leq x),(i=0,1,2,3,4) \\
Q_{i}(t, y)=P\left(S(t)=i, Y_{1}(t) \leq y\right),(i=7,9), Q_{i}(t, z)=P\left(S(t)=i, Y_{2}(t) \leq z\right),(i=8,10)
\end{gathered}
$$

where $P(A)$ is probability of event $A$, and denote:

$$
P_{i}(t, u)=\frac{d}{d u} Q_{i}(t, u)(i=0,1,2,3,4,7,8,9,10)
$$


the following relations are valid:

$$
Q_{i}(t, \infty)=\int_{0}^{\infty} P_{i}(t, u) d u,(i=0,1,2,3,4,7,8,9,10)
$$

where $p_{i}(t)=P(S(t)=i)(i=0,1,2, \ldots, 10)$.

Using the probability arguments and limiting transitions shown in Appendix, we have the following integro-differential equations:

$$
\begin{gathered}
\left(\frac{\partial}{\partial t}+\frac{\partial}{\partial x}+r_{i+1} \lambda+\alpha(x)\right) P_{i}(t, x)=0,(i=0,1) \\
\left(\frac{\partial}{\partial t}+\frac{\partial}{\partial x}+r_{2} \lambda+\alpha(x)\right) P_{2}(t, x)=r_{1} \lambda P_{0}(t, x) \\
\left(\frac{\partial}{\partial t}+\frac{\partial}{\partial x}+r_{1} \lambda+\alpha(x)\right) P_{3}(t, x)=r_{2} \lambda P_{1}(t, x) \\
\left(\frac{\partial}{\partial t}+\frac{\partial}{\partial x}+\alpha(x)\right) P_{4}(t, x)=r_{1} \lambda P_{3}(t, x)+r_{2} \lambda P_{2}(t, x) \\
\left(\frac{d}{d t}+r_{i} \lambda\right) p_{i+4}(t)=\int_{0}^{\infty} P_{i-1}(t, x) \alpha(x) \mathrm{d} x,(i=1,2) \\
\left(\frac{\partial}{\partial t}+\frac{\partial}{\partial y}+r_{2} \lambda+\mu_{1}(y)\right) P_{7}(t, y)=0 \\
\left(\frac{\partial}{\partial t}+\frac{\partial}{\partial z}+r_{1} \lambda+\mu_{2}(z)\right) P_{8}(t, z)=0 \\
\left(\frac{\partial}{\partial t}+\frac{\partial}{\partial y}+\mu_{1}(y)\right) P_{9}(t, y)=r_{2} \lambda P_{7}(t, y) \\
\left(\frac{\partial}{\partial t}+\frac{\partial}{\partial z}+\mu_{2}(z)\right) P_{10}(t, z)=r_{1} \lambda P_{8}(t, z)
\end{gathered}
$$

Their boundary conditions are:

$$
\begin{gathered}
P_{0}(t, 0)=\int_{0}^{\infty} P_{8}(t, z) \mu_{2}(z) \mathrm{d} z+\delta(t), \quad P_{1}(t, 0)=\int_{0}^{\infty} P_{7}(t, y) \mu_{1}(y) \mathrm{d} y \\
P_{i}(t, 0)=0,(i=2,3,4,10), P_{7}(t, 0)=\int_{0}^{\infty} P_{2}(t, x) \alpha(x) \mathrm{d} x+\int_{0}^{\infty} P_{10}(t, z) \mu_{2}(z) \mathrm{d} z+r_{1} \lambda p_{5}(t) \\
P_{8}(t, 0)=\int_{0}^{\infty} P_{3}(t, x) \alpha(x) \mathrm{d} x+\int_{0}^{\infty} P_{9}(t, y) \mu_{1}(y) \mathrm{d} y+r_{2} \lambda p_{6}(t), P_{9}(t, 0)=\int_{0}^{\infty} P_{4}(t, x) \alpha(x) \mathrm{d} x
\end{gathered}
$$


According to the formula of the total probability, we have:

$$
\sum_{i=0}^{4} \int_{0}^{\infty} P_{i}(t, x) \mathrm{d} x+p_{5}(t)+p_{6}(t)+\sum_{i=7}^{10} \int_{0}^{\infty} P_{i}(t, y) \mathrm{d} y=1
$$

The initial conditions are:

$$
P_{0}(0, x)=\delta(x)=\left\{\begin{array}{ll}
1, x=0 ; \\
0, x \neq 0 ;
\end{array}, P_{i}(0, u)=0, u \neq 0,(i=1,2,3,4,7,8,9,10), p_{5}(0)=p_{6}(0)=0 .\right.
$$

\section{Model analysis}

\subsection{Solutions of the equations}

We introduce the Laplace transform and a token as follows:

$$
h^{*}(s)=L_{s}[h(x)]=\int_{0}^{\infty} h(x) e^{-s x} \mathrm{~d} x, s>0
$$

The ergodicity of the investigated process ensures the existence of the following steady-probability: $p_{i}=\lim _{t \rightarrow \infty} p_{i}(t) \quad(i=0,1, \cdots, 10), g_{i}(u)=\lim _{t \rightarrow \infty} P_{i}(t, u) \quad(i=0,1,2,3,4,7,8,9,10)$, which follows the following relations: $p_{i}=\int_{0}^{\infty} g_{i}(u) \mathrm{d} u \quad(i=0,1,2,3,4,7,8,9,10)$.

By taking the limit $t \rightarrow \infty$ in the equations $(1) \sim(13)$, we can obtain the following equations:

$$
\begin{gathered}
\left(\frac{d}{d x}+r_{i+1} \lambda+\alpha(x)\right) g_{i}(x)=0,(i=0,1) \\
\left(\frac{d}{d x}+r_{2} \lambda+\alpha(x)\right) g_{2}(x)=r_{1} \lambda g_{0}(x) \\
\left(\frac{d}{d x}+r_{1} \lambda+\alpha(x)\right) g_{3}(x)=r_{2} \lambda g_{1}(x) \\
\left(\frac{d}{d x}+\alpha(x)\right) g_{4}(x)=r_{1} \lambda g_{3}(x)+r_{2} \lambda g_{2}(x)
\end{gathered}
$$




$$
\begin{gathered}
r_{i} \lambda p_{i+4}=\int_{0}^{\infty} g_{i-1}(x) \alpha(x) \mathrm{d} x,(i=1,2) \\
\left(\frac{d}{d y}+r_{2} \lambda+\mu_{1}(y)\right) g_{7}(y)=0 \\
\left(\frac{d}{d z}+r_{1} \lambda+\mu_{2}(z)\right) g_{8}(z)=0 \\
\left(\frac{d}{d y}+\mu_{1}(y)\right) g_{9}(y)=r_{2} \lambda g_{7}(y) \\
\left(\frac{d}{d z}+\mu_{2}(z)\right) g_{10}(z)=r_{1} \lambda g_{8}(z)
\end{gathered}
$$

The boundary conditions are:

$$
\begin{gathered}
g_{0}(0)=\int_{0}^{\infty} g_{8}(z) \mu_{2}(z) \mathrm{d} z, \quad g_{1}(0)=\int_{0}^{\infty} g_{7}(y) \mu_{1}(y) \mathrm{d} y \\
g_{i}(0)=0,(i=2,3,4,10), \quad g_{7}(0)=\int_{0}^{\infty} g_{2}(x) \alpha(x) \mathrm{d} x+\int_{0}^{\infty} g_{10}(z) \mu_{2}(z) \mathrm{d} z+r_{1} \lambda p_{5} \\
g_{8}(0)=\int_{0}^{\infty} g_{3}(x) \alpha(x) \mathrm{d} x+\int_{0}^{\infty} g_{9}(y) \mu_{1}(y) \mathrm{d} y+r_{2} \lambda p_{6}, \quad g_{9}(0)=\int_{0}^{\infty} g_{4}(x) \alpha(x) \mathrm{d} x \\
\sum_{i=0}^{4} \int_{0}^{\infty} g_{i}(x) \mathrm{d} x+p_{5}+p_{6}+\sum_{i=7}^{10} \int_{0}^{\infty} g_{i}(y) \mathrm{d} y=1
\end{gathered}
$$

We can obtain the solutions $g_{i}(x),(i=0,1,3,4,7,8,9,10), p_{5}, p_{6}$ of the above equations (14) (25) .Follow equation(26), we can find $c_{0}$ :

$$
\begin{aligned}
c_{0}^{-1}=\frac{1}{\alpha} & +\frac{v^{*}\left(r_{1} \lambda\right)}{r_{1} \lambda}+\frac{1}{\mu_{2} h_{2}^{*}\left(r_{1} \lambda\right)}+\left(\frac{h_{1}^{*}\left(r_{2} \lambda\right)}{\alpha}+\frac{h_{1}^{*}\left(r_{2} \lambda\right) v^{*}\left(r_{2} \lambda\right)}{r_{2} \lambda}\right)\left(\frac{1}{h_{2}^{*}\left(r_{1} \lambda\right)}-\frac{r_{1}\left(1-v^{*}\left(r_{2} \lambda\right)\right)-r_{2}\left(1-v^{*}\left(r_{1} \lambda\right)\right)}{r_{1}-r_{2}}\right) \\
& +\frac{1}{\mu_{1}}\left(\frac{1}{h_{2}^{*}\left(r_{1} \lambda\right)}+\frac{\left(r_{1}\left(1-v^{*}\left(r_{2} \lambda\right)\right)-r_{2}\left(1-v^{*}\left(r_{1} \lambda\right)\right)\right) h_{1}^{*}\left(r_{2} \lambda\right)}{\left(r_{1}-r_{2}\right) h_{2}^{*}\left(r_{1} \lambda\right)}-\frac{\left(r_{1}\left(1-v^{*}\left(r_{2} \lambda\right)\right)-r_{2}\left(1-v^{*}\left(r_{1} \lambda\right)\right)\right)^{2} h_{1}^{*}\left(r_{2} \lambda\right)}{\left(r_{1}-r_{2}\right)^{2}}\right)
\end{aligned}
$$

From $p_{i}=\int_{0}^{\infty} g_{i}(u) \mathrm{d} u \quad(i=0,1,2,3,4,7,8,9,10)$, We can obtain the following steady-state probability:

$$
\begin{gathered}
p_{0}=V^{*}\left(r_{1} \lambda\right) c_{0}, \quad p_{1}=\left(\frac{1}{h_{2}^{*}\left(r_{1} \lambda\right)}-\frac{r_{1}\left(1-v^{*}\left(r_{2} \lambda\right)\right)-r_{2}\left(1-v^{*}\left(r_{1} \lambda\right)\right.}{r_{1}-r_{2}}\right) h_{1}^{*}\left(r_{2} \lambda\right) V^{*}\left(r_{2} \lambda\right) c_{0} \\
p_{2}=\frac{r_{1}\left(V^{*}\left(r_{2} \lambda\right)-V^{*}\left(r_{1} \lambda\right)\right)}{r_{1}-r_{2}} c_{0}, \quad p_{10}=\left(\frac{1}{\mu_{2} h_{2}^{*}\left(r_{1} \lambda\right)}-\frac{\bar{H}_{2}^{*}\left(r_{1} \lambda\right)}{h_{2}^{*}\left(r_{1} \lambda\right)}\right) c_{0}
\end{gathered}
$$




$$
\begin{aligned}
& p_{3}=\left.\frac{r_{2}\left(V^{*}\left(r_{2} \lambda\right)-V^{*}\left(r_{1} \lambda\right)\right)}{\left(r_{1}-r_{2}\right) h_{2}^{*}\left(r_{1} \lambda\right)}-\frac{r_{2}\left(V^{*}\left(r_{2} \lambda\right)-V^{*}\left(r_{1} \lambda\right)\right)\left(r_{1}\left(1-v^{*}\left(r_{2} \lambda\right)\right)-r_{2}\left(1-v^{*}\left(r_{1} \lambda\right)\right)\right)}{\left(r_{1}-r_{2}\right)^{2}}\right) h_{1}^{*}\left(r_{2} \lambda\right) c_{0} \\
& p_{4}= \frac{\left(h_{1}^{*}\left(r_{2} \lambda\right)+h_{2}^{*}\left(r_{1} \lambda\right)\right)\left(r_{2} V^{*}\left(r_{1} \lambda\right)-r_{1} V^{*}\left(r_{2} \lambda\right)\right)}{\left(r_{1}-r_{2}\right) h_{2}^{*}\left(r_{1} \lambda\right)} c_{0}-\frac{\left[r_{1}\left(1-v^{*}\left(r_{2} \lambda\right)\right)-r_{2}\left(1-v^{*}\left(r_{1} \lambda\right)\right)\right] h_{1}^{*}\left(r_{2} \lambda\right)}{\alpha\left(r_{1}-r_{2}\right)} c_{0} \\
&+\frac{h_{1}^{*}\left(r_{2} \lambda\right)+h_{2}^{*}\left(r_{1} \lambda\right)}{\alpha h_{2}^{*}\left(r_{1} \lambda\right)} c_{0}-\frac{\left(r_{2} V^{*}\left(r_{1} \lambda\right)-r_{1} V^{*}\left(r_{2} \lambda\right)\right)\left(r_{1}\left(1-v^{*}\left(r_{2} \lambda\right)\right)-r_{2}\left(1-v^{*}\left(r_{1} \lambda\right)\right)\right) h_{1}^{*}\left(r_{2} \lambda\right)}{\left(r_{1}-r_{2}\right)^{2}} c_{0} \\
& p_{5}= \frac{v^{*}\left(r_{1} \lambda\right)}{r_{1} \lambda} c_{0}, \quad p_{6}=\left(\frac{v^{*}\left(r_{2} \lambda\right) h_{1}^{*}\left(r_{2} \lambda\right)}{r_{2} \lambda h_{2}^{*}\left(r_{1} \lambda\right)}-\frac{\left(r_{1}\left(1-v^{*}\left(r_{2} \lambda\right)\right)-r_{2}\left(1-v^{*}\left(r_{1} \lambda\right)\right)\right) h_{1}^{*}\left(r_{2} \lambda\right) v^{*}\left(r_{2} \lambda\right)}{r_{2} \lambda\left(r_{1}-r_{2}\right)}\right) c_{0} \\
& p_{7}=\left(\frac{\bar{H}_{1}^{*}\left(r_{2} \lambda\right)}{h_{2}^{*}\left(r_{1} \lambda\right)}-\frac{\left(r_{1}\left(1-v^{*}\left(r_{2} \lambda\right)\right)-r_{2}\left(1-v^{*}\left(r_{1} \lambda\right)\right)\right) \bar{H}_{1}^{*}\left(r_{2} \lambda\right)}{r_{1}-r_{2}}\right) c_{2}^{*}\left(r_{1} \lambda\right) p_{0} \\
& p_{9}= \frac{1}{\mu_{1}}\left(\frac{1}{h_{2}^{*}\left(r_{1} \lambda\right)}+\frac{\left(r_{1}\left(1-v^{*}\left(r_{2} \lambda\right)\right)-r_{2}\left(1-v^{*}\left(r_{1} \lambda\right)\right)\right) h_{1}^{*}\left(r_{2} \lambda\right)}{\left(r_{1}-r_{2}\right) h_{2}^{*}\left(r_{1} \lambda\right)}-\frac{\left(r_{1}\left(1-v^{*}\left(r_{2} \lambda\right)\right)-r_{2}\left(1-v^{*}\left(r_{1} \lambda\right)\right)\right)^{2} h_{1}^{*}\left(r_{2} \lambda\right)}{\left(r_{1} r_{2}\right.}\right) c_{0} \\
&-\left(\frac{\bar{H}_{1}^{*}\left(r_{2} \lambda\right)}{h_{2}^{*}\left(r_{1} \lambda\right)}-\frac{\left(r_{1}\left(1-v^{*}\left(r_{2} \lambda\right)\right)-r_{2}\left(1-v^{*}\left(r_{1} \lambda\right)\right)\right) \bar{H}_{1}^{*}\left(r_{2} \lambda\right)}{r_{1}-r_{2}}\right) c_{0}
\end{aligned}
$$

\subsection{Reliability indices}

Hence, the following results are obtained. The steady-state availability of the system (see[13], for example) is

$$
\begin{aligned}
A= & p_{0}+p_{1}+p_{2}+p_{3}+p_{5}+p_{6}+p_{7}+p_{8} \\
= & \left(\frac{r_{1} V^{*}\left(r_{2} \lambda\right)-r_{2} V^{*}\left(r_{1} \lambda\right)}{r_{1}-r_{2}}+\frac{v^{*}\left(r_{1} \lambda\right)}{r_{1} \lambda}\right) c_{0}++\left(\frac{\bar{H}_{1}^{*}\left(r_{2} \lambda\right)+\bar{H}_{2}^{*}\left(r_{1} \lambda\right)}{h_{2}^{*}\left(r_{1} \lambda\right)}-\frac{\left(r_{1}\left(1-v^{*}\left(r_{2} \lambda\right)\right)-r_{2}\left(1-v^{*}\left(r_{1} \lambda\right)\right)\right) \bar{H}_{1}^{*}\left(r_{2} \lambda\right)}{r_{1}-r_{2}}\right) c_{0} \\
& +\left(\frac{r_{1} V^{*}\left(r_{2} \lambda\right)-r_{2} V^{*}\left(r_{1} \lambda\right)}{r_{1}-r_{2}}+\frac{v^{*}\left(r_{2} \lambda\right)}{r_{2} \lambda}\right)\left(\frac{h_{1}^{*}\left(r_{2} \lambda\right)}{h_{2}^{*}\left(r_{1} \lambda\right)}-\frac{\left(r_{1}\left(1-v^{*}\left(r_{2} \lambda\right)\right)-r_{2}\left(1-v^{*}\left(r_{1} \lambda\right)\right)\right) h_{1}^{*}\left(r_{2} \lambda\right)}{r_{1}-r_{2}}\right) c_{0}
\end{aligned}
$$

The steady state probability that the repairman is on vacation is

$$
P_{V}=p_{0}+p_{1}+p_{2}+p_{3}+p_{4}=\left(\frac{h_{1}^{*}\left(r_{2} \lambda\right)+h_{2}^{*}\left(r_{1} \lambda\right)}{\alpha h_{2}^{*}\left(r_{1} \lambda\right)}-\frac{\left(r_{1}\left(1-v^{*}\left(r_{2} \lambda\right)\right)-r_{2}\left(1-v^{*}\left(r_{1} \lambda\right)\right)\right) h_{1}^{*}\left(r_{2} \lambda\right)}{\alpha\left(r_{1}-r_{2}\right)}\right) c_{0}
$$

The probability that the system is waiting for repair, namely, the probability that the system is in failure but the repairman is on vacation is $P_{w}(t)$. From assumptions of the system, we have $P_{w}(t)=\int_{0}^{\infty} P_{4}(t, x) \mathrm{d} x$, and the steady state probability that the system is waiting for being repaired is $P_{w}=p_{4}$

Following the result of [13], the steady-state failure frequency of the system is given by 


$$
\begin{aligned}
M & =r_{2} \lambda p_{2}+r_{1} \lambda p_{3}+r_{2} \lambda p_{7}+r_{1} \lambda p_{8} \\
& =\frac{r_{1} r_{2} \lambda\left(\bar{V}^{*}\left(r_{2} \lambda\right)-\bar{V}^{*}\left(r_{1} \lambda\right)\right)}{r_{1}-r_{2}} \frac{h_{1}^{*}\left(r_{2} \lambda\right)+h_{2}^{*}\left(r_{1} \lambda\right)}{h_{2}^{*}\left(r_{1} \lambda\right)} c_{0}-\frac{r_{1} r_{2} \lambda\left(\bar{V}^{*}\left(r_{2} \lambda\right)-\bar{V}^{*}\left(r_{1} \lambda\right)\right)}{r_{1}-r_{2}} \frac{\left(r_{1}\left(1-v^{*}\left(r_{2} \lambda\right)\right)-r_{2}\left(1-v^{*}\left(r_{1} \lambda\right)\right)\right) h_{1}^{*}\left(r_{2} \lambda\right)}{r_{1}-r_{2}} c_{0} \\
& +\left(\frac{r_{2} \lambda \bar{H}_{1}^{*}\left(r_{2} \lambda\right)+r_{1} \lambda \bar{H}_{2}^{*}\left(r_{1} \lambda\right)}{h_{2}^{*}\left(r_{1} \lambda\right)}-\frac{\left(r_{1}\left(1-v^{*}\left(r_{2} \lambda\right)\right)-r_{2}\left(1-v^{*}\left(r_{1} \lambda\right)\right)\right) r_{2} \lambda \bar{H}_{1}^{*}\left(r_{2} \lambda\right)}{r_{1}-r_{2}}\right) c_{0}
\end{aligned}
$$

The mean up-time is expressed in terms of the steady-state probability of the system (see [16]) by MUT: $M U T=\frac{A}{M}$.

In order to obtain system reliability, we let the above three failure states 4,9 and 10 be the absorbing states, then we have $\left\{\left(\widetilde{S}(t), \widetilde{X}(t), \widetilde{Y}_{1}(t), \widetilde{Y}_{2}(t)\right), t \geq 0\right\}$. Let $\mathcal{Q}_{i}(t, x)=\frac{d}{d x} P(\widetilde{S}(t)=i, \widetilde{X}(t) \leq$ $x),(i=0,1,2,3), \mathcal{Q}_{i}(t)=P(\widetilde{S}(t)=i),(i=5,6) \mathcal{Q}_{7}(t, y)=\frac{d}{d y} P\left(\widetilde{S}(t)=7, \widetilde{Y}_{1}(t) \leq y\right), \mathcal{Q}_{8}(t, z)=$ $\frac{d}{d z} P\left(\widetilde{S}(t)=8, \widetilde{Y}_{2}(t) \leq z\right)$.

Using the method similar to that in section 3, we have the following partial-differential equations:

$$
\begin{gathered}
\left(\frac{\partial}{\partial t}+\frac{\partial}{\partial x}+r_{i+1} \lambda+\alpha(x)\right) \mathcal{Q}_{i}(t, x)=0,(i=0,1) \\
\left(\frac{\partial}{\partial t}+\frac{\partial}{\partial x}+r_{2} \lambda+\alpha(x)\right) \mathcal{Q}_{2}(t, x)=r_{1} \lambda \mathcal{Q}_{0}(t, x) \\
\left(\frac{\partial}{\partial t}+\frac{\partial}{\partial x}+r_{1} \lambda+\alpha(x)\right) \mathcal{Q}_{3}(t, x)=r_{2} \lambda \mathcal{Q}_{1}(t, x) \\
\left(\frac{d}{d t}+r_{i} \lambda\right) \mathcal{Q}_{i+4}(t)=\int_{0}^{\infty} \mathcal{Q}_{i-1}(t, x) \alpha(x) \mathrm{d} x,(i=1,2) \\
\left(\frac{\partial}{\partial t}+\frac{\partial}{\partial y}+r_{2} \lambda+\mu_{1}(y)\right) \mathcal{Q}_{7}(t, y)=0 \\
\left(\frac{\partial}{\partial t}+\frac{\partial}{\partial z}+r_{1} \lambda+\mu_{2}(z)\right) \mathcal{Q}_{8}(t, z)=0
\end{gathered}
$$

The boundary conditions are:

$$
\begin{gathered}
\mathcal{Q}_{0}(t, 0)=\int_{0}^{\infty} \mathcal{Q}_{8}(t, z) \mu_{2}(z) \mathrm{d} z+\delta(t), \mathcal{Q}_{1}(t, 0)=\int_{0}^{\infty} \mathcal{Q}_{7}(t, y) \mu_{1}(y) \mathrm{d} y, \mathcal{Q}_{i}(t, 0)=0,(i=2,3) \\
\mathcal{Q}_{7}(t, 0)=\int_{0}^{\infty} \mathcal{Q}_{2}(t, x) \alpha(x) \mathrm{d} x+r_{1} \lambda \mathcal{Q}_{5}(t), \quad \mathcal{Q}_{8}(t, 0)=\int_{0}^{\infty} \mathcal{Q}_{3}(t, x) \alpha(x) \mathrm{d} x+r_{2} \lambda \mathcal{Q}_{6}(t)
\end{gathered}
$$


The initial conditions are:

$$
\mathcal{Q}_{0}(0, x)=\delta(x)=\left\{\begin{array}{c}
1, x=0 \\
0, x \neq 0
\end{array}\right.
$$

otherwise is 0 .

Theorem 1 The Laplace transformation formula of the reliability of the system is given by

$$
\begin{aligned}
R^{*}(s)= & \frac{\left(r_{1} V^{*}\left(s+r_{2} \lambda\right)-r_{2} V^{*}\left(s+r_{1} \lambda\right)\right)\left(1+C_{1}(s) h_{1}^{*}\left(s+r_{2} \lambda\right)\right)}{\left(r_{1}-r_{2}\right)\left(1-C_{1}(s) C_{2}(s) h_{1}^{*}\left(s+r_{2} \lambda\right) h_{2}^{*}\left(s+r_{1} \lambda\right)\right)}+\frac{C_{1}(s) \bar{H}_{1}^{*}\left(s+r_{2} \lambda\right)+C_{1}(s) C_{2}(s) \bar{H}_{2}^{*}\left(s+r_{1} \lambda\right) h_{1}^{*}\left(s+r_{2} \lambda\right)}{1-C_{1}(s) C_{2}(s) h_{1}^{*}\left(s+r_{2} \lambda\right) h_{2}^{*}\left(s+r_{1} \lambda\right)} \\
& +\left(\frac{v^{*}\left(s+r_{1} \lambda\right)}{s+r_{1} \lambda}+\frac{C_{1}(s) h_{1}^{*}\left(s+r_{2} \lambda\right) v^{*}\left(s+r_{2} \lambda\right)}{s+r_{2} \lambda}\right) \frac{1}{1-C_{1}(s) C_{2}(s) h_{1}^{*}\left(s+r_{2} \lambda\right) h_{2}^{*}\left(s+r_{1} \lambda\right)}
\end{aligned}
$$

where: $C_{i}(s)=\frac{r_{i} v^{*}\left(s+r_{2} \lambda\right)-r_{i} v^{*}\left(s+r_{1} \lambda\right)}{r_{1}-r_{2}}+\frac{r_{i} \lambda v^{*}\left(s+r_{i} \lambda\right)}{s+r_{i} \lambda},(i=1,2)$

The Proof of Theorem 1 is given in the Appendix

Corollary 1 The mean time to the first failure of the system is:

$$
\begin{aligned}
\operatorname{MTTFF}= & \frac{\left(r_{1} V^{*}\left(r_{2} \lambda\right)-r_{2} V^{*}\left(r_{1} \lambda\right)\right)\left(r_{1}-r_{2}+\left(r_{1} v^{*}\left(r_{2} \lambda\right)-r_{2} v^{*}\left(r_{1} \lambda\right)\right) h_{1}^{*}\left(r_{2} \lambda\right)\right)}{\left.\left(r_{1}-r_{2}\right)^{2}-\left(r_{1} v^{*}\left(r_{2} \lambda\right)-r_{2} v^{*}\left(r_{1} \lambda\right)\right)^{2} h_{1}^{*}\left(r_{2} \lambda\right) h_{2}^{*}\left(r_{1} \lambda\right)\right)} \\
& +\frac{r_{2}\left(r_{1}-r_{2}\right)^{2} v^{*}\left(r_{1} \lambda\right)+r_{1}\left(r_{1}-r_{2}\right) v^{*}\left(r_{2} \lambda\right) h_{1}^{*}\left(r_{2} \lambda\right)\left(r_{1} v^{*}\left(r_{2} \lambda\right)-r_{2} v^{*}\left(r_{1} \lambda\right)\right)}{r_{1} r_{2} \lambda\left(\left(r_{1}-r_{2}\right)^{2}-\left(r_{1} v^{*}\left(r_{2} \lambda\right)-r_{2} v^{*}\left(r_{1} \lambda\right)\right)^{2} h_{1}^{*}\left(r_{2} \lambda\right) h_{2}^{*}\left(r_{1} \lambda\right)\right)} \\
& +\frac{\left(r_{1}-r_{2}\right) \bar{H}_{1}^{*}\left(r_{2} \lambda\right)\left(r_{1} v^{*}\left(r_{2} \lambda\right)-r_{2} v^{*}\left(r_{1} \lambda\right)\right)+\bar{H}_{2}^{*}\left(r_{1} \lambda\right) h_{1}^{*}\left(r_{2} \lambda\right)\left(r_{1} v^{*}\left(r_{2} \lambda\right)-r_{2} v^{*}\left(r_{1} \lambda\right)\right)^{2}}{\left(r_{1}-r_{2}\right)^{2}-\left(r_{1} v^{*}\left(r_{2} \lambda\right)-r_{2} v^{*}\left(r_{1} \lambda\right)\right)^{2} h_{1}^{*}\left(r_{2} \lambda\right) h_{2}^{*}\left(r_{1} \lambda\right)}
\end{aligned}
$$

Proof Calculating MTTFF $=\int_{0}^{\infty} R(t) \mathrm{d} t=\lim _{s \rightarrow 0^{+}} R^{*}(s)$ implies the result.

\section{Special case}

In this section, we discuss the following special cases, which verify the results of the preceding section.

Case 1. If $r_{1}=r_{2}=r=0$, then it implies that shocks do no harm on the working unit and the units will never fail;

Case 2. If $r_{1}=r_{2}=r=1$, then each shock will cause the working unit to fail and the repairman will take vacations; 
Case 3. If $r_{1}=r_{2}=r=1$, and $P(Z=0)=1$, then each shock will cause the working unit to fail and the repairman will repair once the unit fails.

Corresponding results can be easily obtained for the above special cases.

\section{$6 \quad$ Numerical examples}

To validate the above derivation, we conduct the following numerical experiment. Here, we assume

$$
F(x)=\left\{\begin{array}{l}
1-e^{-\eta x}, x>0 ; \\
0, \quad x \leq 0 ;
\end{array}, \Phi_{1}(x)=\left\{\begin{array}{c}
1-e^{-\frac{\eta}{4} x}, x>0 ; \\
0, \quad x \leq 0 ;
\end{array}, \Phi_{2}(x)=\left\{\begin{array}{l}
1-e^{-\frac{\eta}{3} x}, x>0 ; \\
0,
\end{array} .\right.\right.\right.
$$

Then we have

$$
\begin{aligned}
& r_{1}=P\left(\widehat{X}>\tau_{1}\right)=\int_{0}^{\infty} P\left(\tau_{1}<\widehat{x} \mid \widehat{X}=\widehat{x}\right) \mathrm{d} P(\widehat{X} \leq \widehat{x})=\int_{0}^{\infty}\left(1-e^{-\frac{\eta}{4} \widehat{x}}\right) \mathrm{d}\left(1-e^{-\eta \widehat{x}}\right)=\frac{1}{5}, \\
& r_{2}=P\left(\widehat{X}>\tau_{2}\right)=\int_{0}^{\infty} P\left(\tau_{2}<\widehat{x} \mid \widehat{X}=\widehat{x}\right) \mathrm{d} P(\widehat{X} \leq \widehat{x})=\int_{0}^{\infty}\left(1-e^{-\frac{\eta}{3} \widehat{x}}\right) \mathrm{d}\left(1-e^{-\eta \widehat{x}}\right)=\frac{1}{4},
\end{aligned}
$$

We assume the repair time distribution of the unit $i(\mathrm{i}=1,2)$ and the vacation time distribution of the repairman are exponential distributions, i.e. $h_{i}(t)=\mu_{i} \exp \left(-\mu_{i} t\right) \quad(i=1,2)$ and $v(t)=$ $\alpha \exp (-\alpha t)$

We first present numerical examples comparing the reliability indices for the situations when the repairman is assumed to have single vacation and no vacation. Figures 1, 2 and 3 shows the steady-state probability of the repairman vacation and the steady-state probability of wait-forrepair time when a single vacation of the repairman is assumed. From the curves of Figs. 1, 2, and 3 , we conclude that the steady-state availability $A$, mean up-time $M U T$, and mean time to the first failure for the model with single vacation increase uniformly. They increase rapidly at an early stage and then stable as $\alpha$ becomes larger. The curves of Fig. 4 and Fig. 5 shows that, when the repairman only takes single vacation, both the steady-state probability $P_{V}$ of the length of the vacation and the steady-state probability $P_{w}$ of system's waiting-for-repair time decrease as 
the rate $\alpha$ increases. The decrease is rapid initially and then becomes stable as $\alpha$ becomes larger. Fig. 6 shows the steady-state availability increases as $\alpha$ increases. It can also be observed from Fig. 6 that the intensity values $\lambda$ affect the steady-state availability significantly, the steady-state availability decreases as intensity $\lambda$ increases.

The mean up-time MUT and the mean time to the first failure MTTFF are investigated, when $\lambda$ and $\alpha$ change, as shown in Table 1 and Table 2 . We change the values of $\lambda$ and $\alpha$ and observe their cross effects on the mean up-time MUT and the mean time. It shows that increasing $\lambda$ can significantly decrease the MUT and the MTTFF, however, increasing $\alpha$ rarely affects the values of MUT and MTTFF.

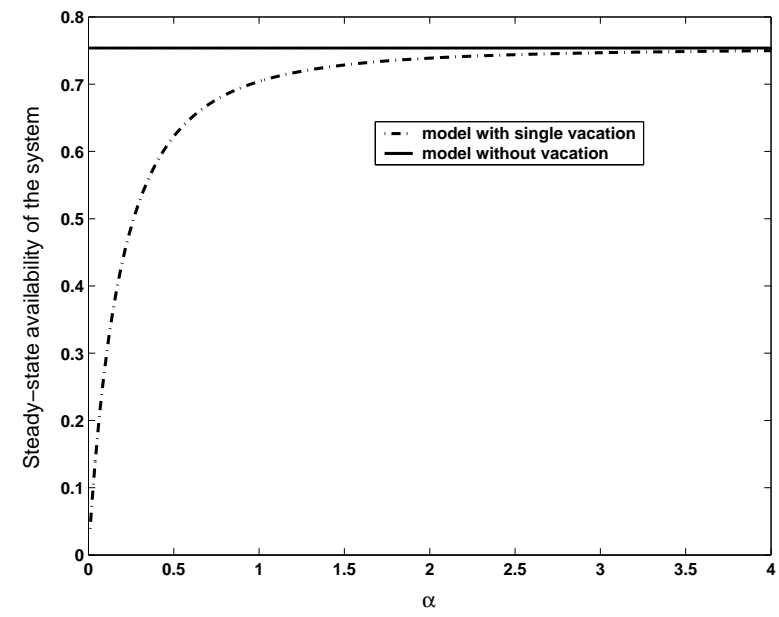

Figure 1. Steady-state availability versus rate $\alpha$ when $\lambda=3.0, \mu_{1}=0.8, \mu_{2}=1.0$.

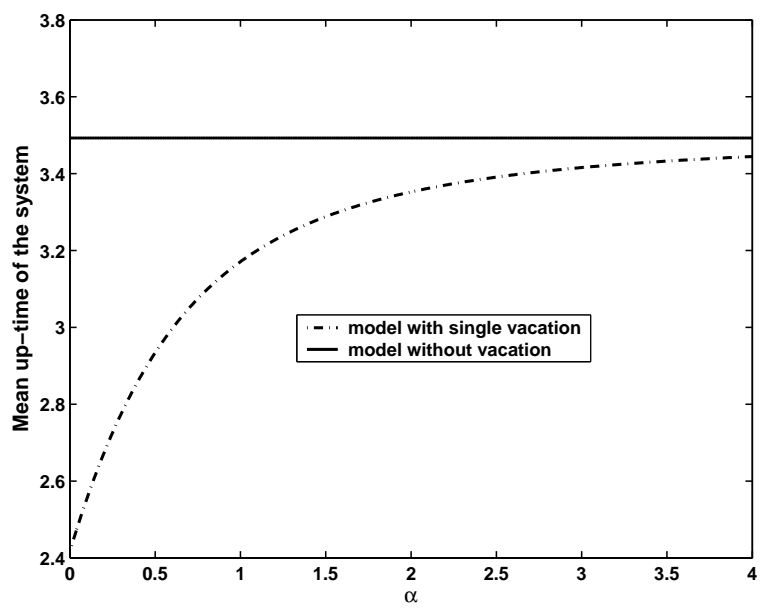

Figure 2. Mean up-time versus rate $\alpha$ when $\lambda=3.0, \mu_{1}=0.8, \mu_{2}=1.0$. 


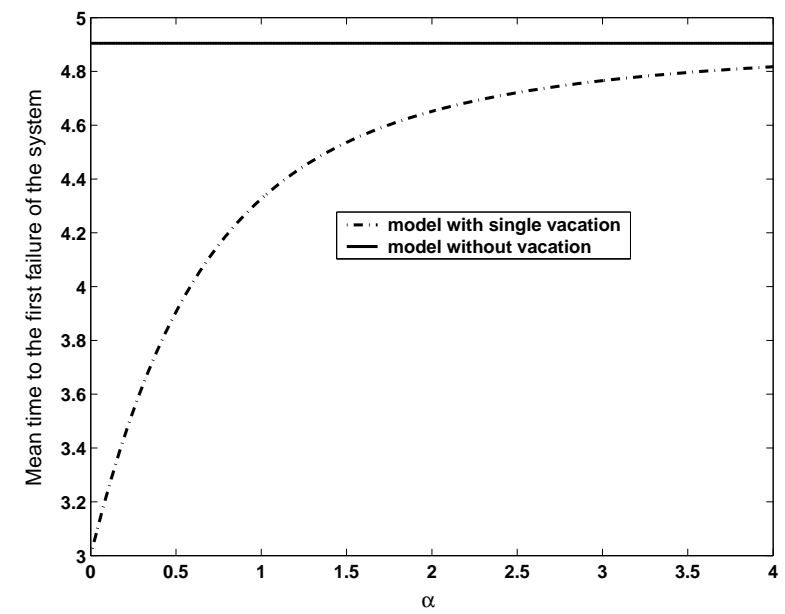

Figure 3. Mean time to the first failure versus rate $\alpha$ when $\lambda=3.0, \mu_{1}=0.8, \mu_{2}=1.0$.

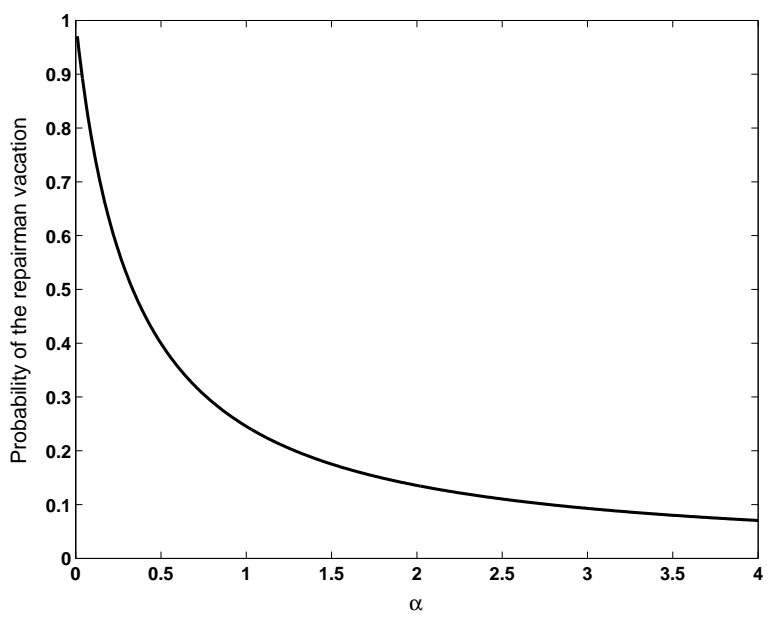

Figure 4. Steady-state probability of the repairman vacation versus rate $\alpha$ when

$$
\lambda=3.0, \mu_{1}=0.8, \mu_{2}=1.0 .
$$

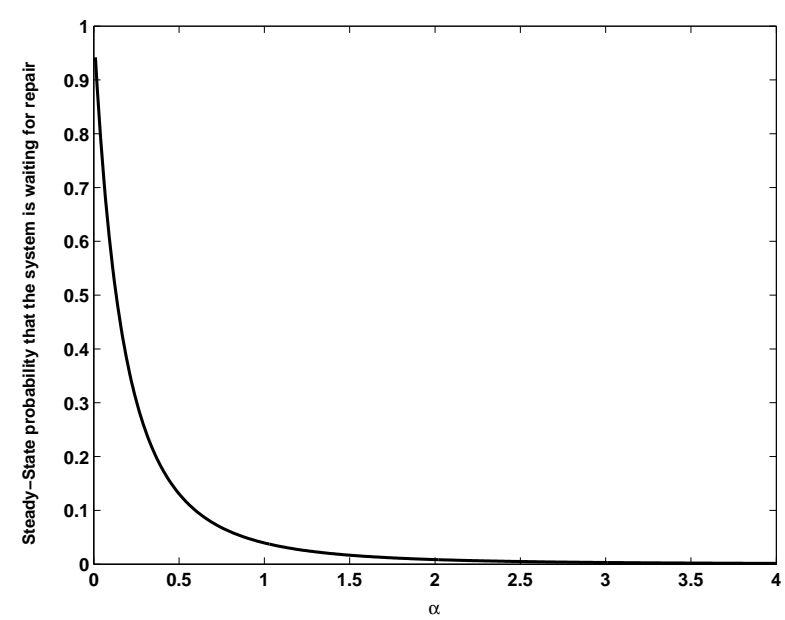


Figure 5. Steady-state probability that the system is waiting for repair versus rate $\alpha$ when

$$
\lambda=3.0, \mu_{1}=0.8, \mu_{2}=1.0 .
$$

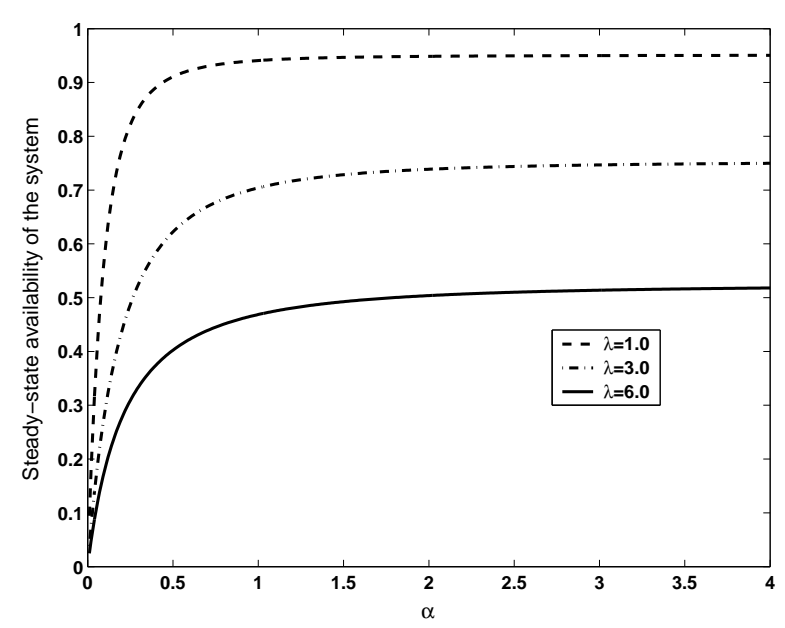

Figure 6. Steady-state availability versus rate $\alpha$ for different intensity values of the Poisson shock process when $\mu_{1}=0.8, \mu_{2}=1.0$.

Table 1. Mean up-time for different $\alpha$ and intensity values of the Poisson shock process when

$$
\mu_{1}=0.8, \mu_{2}=1.0 .
$$

\begin{tabular}{|c|c|c|c|c|c|c|c|c|}
\hline$\lambda$ & $\alpha=1$ & $\alpha=5$ & $\alpha=9$ & $\alpha=13$ & $\alpha=17$ & $\alpha=21$ & $\alpha=25$ & $\alpha=29$ \\
\hline 2.0 & 6.0465 & 6.6581 & 6.6947 & 6.7040 & 6.7077 & 6.7095 & 6.7106 & 6.7112 \\
2.2 & 5.5123 & 5.7027 & 5.7243 & 5.7308 & 5.7335 & 5.7349 & 5.7358 & 5.7363 \\
2.4 & 4.4960 & 4.9368 & 4.9658 & 4.9733 & 4.9763 & 4.9778 & 4.9787 & 4.9792 \\
2.6 & 3.9599 & 4.3398 & 4.3659 & 4.3728 & 4.3755 & 4.3769 & 4.3777 & 4.3781 \\
2.8 & 3.5267 & 3.8569 & 3.8806 & 3.8868 & 3.8893 & 3.8906 & 3.8913 & 3.8918 \\
3.0 & 3.1709 & 3.4599 & 3.4814 & 3.4872 & 3.4895 & 3.4907 & 3.4913 & 3.4917 \\
3.2 & 2.8743 & 3.1288 & 3.1486 & 3.1539 & 3.1560 & 3.1571 & 3.1577 & 3.1581 \\
\hline
\end{tabular}

Table 2. Mean time to the first failure for different $\alpha$ and intensity values of the Poisson shock process 
when $\mu_{1}=0.8, \mu_{2}=1.0$.

\begin{tabular}{|c|c|c|c|c|c|c|c|c|}
\hline$\lambda$ & $\alpha=1$ & $\alpha=5$ & $\alpha=9$ & $\alpha=13$ & $\alpha=17$ & $\alpha=21$ & $\alpha=25$ & $\alpha=29$ \\
\hline 2.0 & 7.7984 & 8.7297 & 8.7855 & 8.7998 & 8.8054 & 8.8082 & 8.8098 & 8.8108 \\
2.2 & 6.7594 & 7.5735 & 7.6248 & 7.6380 & 7.6432 & 7.6459 & 7.6473 & 7.6483 \\
2.4 & 5.9465 & 6.6654 & 6.7129 & 6.7252 & 6.7301 & 6.7326 & 6.7340 & 6.7349 \\
2.6 & 5.2961 & 5.9364 & 5.9806 & 5.9922 & 5.9968 & 5.9991 & 6.0005 & 6.0013 \\
2.8 & 4.7658 & 5.3402 & 5.3816 & 5.3926 & 5.3970 & 5.3992 & 5.4004 & 5.4012 \\
3.0 & 4.3263 & 4.8450 & 4.8840 & 4.8943 & 4.8985 & 4.9006 & 4.9018 & 4.9025 \\
3.2 & 3.9570 & 4.4280 & 4.4648 & 4.4747 & 4.4786 & 4.4807 & 4.4818 & 4.4825 \\
\hline
\end{tabular}

\section{Conclusions and future work}

In this paper, we derived the reliability indices of a system consisting of two different units, a switch and a repairman. The repairman might take vacation and the operating unit might be attacked by shocks. Such a system can be seen as an extension of a general cold standby repairable system, which is one of important repaired systems in the reliability engineering and is also difficult to analyse as there are many random variables with general distributions involved. The numerical data experiments show the relationship between the derived reliability indices and relevant parameters.

In this paper, it is also assumed that if there is no failed unit when the repairman returns from a vacation, he does not take a vacation again and remain idle until the first failed unit appears. This assumption will be relaxed in our future work. 


\section{Appendix}

The derivation of Eqs. $(1) \sim(12)$

Since the process $\left\{\left(S(t), X(t), Y_{1}(t), Y_{2}(t)\right), t \geq 0\right\}$ is a continuous vector Markov process, we can express the process in a way considering the transitions occurring in $t$ and $t+\Delta t$. Relating the state of the system at $t$ and $t+\Delta t$, we readily set up the following partial differential equations (see [17]):

$P_{0}(t+\Delta t, x+\Delta t)=P_{0}(t, x)\left(1-\left(r_{1} \lambda+\alpha(x)\right) \Delta t\right)+o(\Delta t)$,

$P_{1}(t+\Delta t, x+\Delta t)=P_{1}(t, x)\left(1-\left(r_{2} \lambda+\alpha(x)\right) \Delta t\right)+o(\Delta t)$,

$P_{2}(t+\Delta t, x+\Delta t)=P_{2}(t, x)\left(1-\left(r_{2} \lambda+\alpha(x)\right) \Delta t\right)+r_{1} \lambda P_{0}(t, x) \Delta t+o(\Delta t)$,

$P_{3}(t+\Delta t, x+\Delta t)=P_{3}(t, x)\left(1-\left(r_{1} \lambda+\alpha(x)\right) \Delta t\right)+r_{2} \lambda P_{1}(t, x) \Delta t+o(\Delta t)$,

$P_{4}(t+\Delta t, x+\Delta t)=P_{4}(t, x)(1-\alpha(x) \Delta t)+r_{1} \lambda P_{3}(t, x) \Delta t+r_{2} \lambda P_{2}(t, x) \Delta t+o(\Delta t)$,

$p_{5}(t+\Delta t)=p_{5}(t, x)\left(1-r_{1} \lambda \Delta t\right)+\int_{0}^{\infty} P_{0}(t, x) \alpha(x) \mathrm{d} x \Delta t+o(\Delta t)$,

$p_{6}(t+\Delta t)=p_{6}(t, x)\left(1-r_{2} \lambda \Delta t\right)+\int_{0}^{\infty} P_{1}(t, x) \alpha(x) \mathrm{d} x \Delta t+o(\Delta t)$,

$P_{7}(t+\Delta t, y+\Delta t)=P_{7}(t, y)\left(1-\left(r_{2} \lambda+\mu_{1}(y)\right) \Delta t\right)+o(\Delta t)$,

$P_{8}(t+\Delta t, z+\Delta t)=P_{8}(t, z)\left(1-\left(r_{1} \lambda+\mu_{2}(z)\right) \Delta t\right)+o(\Delta t)$,

$P_{9}(t+\Delta t, y+\Delta t)=P_{9}(t, y)\left(1-\mu_{1}(y) \Delta t\right)+r_{2} \lambda P_{7}(t, y) \Delta t+o(\Delta t)$,

$P_{10}(t+\Delta t, z+\Delta t)=P_{10}(t, z)\left(1-\mu_{2}(z) \Delta t\right)+r_{1} \lambda P_{8}(t, z) \Delta t+o(\Delta t)$,

$P_{0}(t+\Delta t, 0) \Delta t=\int_{0}^{\Delta t} P_{0}(t+\Delta t, z) \mathrm{d} z+o(\Delta t)=\int_{0}^{\infty} P_{8}(t, z) \mu_{2}(z) \mathrm{d} z \Delta t+\delta(t) \Delta t+o(\Delta t)$,

$P_{1}(t+\Delta t, 0) \Delta t=\int_{0}^{\Delta t} P_{1}(t+\Delta t, z) \mathrm{d} z+o(\Delta t)=\int_{0}^{\infty} P_{7}(t, y) \mu_{2}(y) \mathrm{d} z \Delta t+o(\Delta t)$,

$P_{2}(t+\Delta t, 0) \Delta t=P_{3}(t+\Delta t, 0) \Delta t=P_{4}(t+\Delta t, 0) \Delta t=P_{10}(t+\Delta t, 0) \Delta t=o(\Delta t)$

$P_{7}(t+\Delta t, 0) \Delta t=\int_{0}^{\Delta t} P_{7}(t+\Delta t, z) \mathrm{d} z+o(\Delta t)=\int_{0}^{\infty} P_{2}(t, x) \alpha(x) \mathrm{d} x \Delta t+\int_{0}^{\infty} P_{10}(t, z) \mu_{2}(z) \mathrm{d} z \Delta t+r_{1} \lambda p_{5}(t) \Delta t+$ $o(\Delta t)$,

$P_{8}(t+\Delta t, 0) \Delta t=\int_{0}^{\Delta t} P_{8}(t+\Delta t, z) \mathrm{d} z+o(\Delta t)=\int_{0}^{\infty} P_{3}(t, x) \alpha(x) \mathrm{d} x \Delta t+\int_{0}^{\infty} P_{9}(t, y) \mu_{1}(y) \mathrm{d} y \Delta t+r_{2} \lambda p_{6}(t) \Delta t+$ $o(\Delta t)$,

$P_{9}(t+\Delta t, 0) \Delta t=\int_{0}^{\Delta t} P_{9}(t+\Delta t, z) \mathrm{d} z+o(\Delta t)=\int_{0}^{\infty} P_{4}(t, x) \alpha(x) \mathrm{d} x \Delta t+o(\Delta t)$,

\section{The proof of Theorem 1 is as follows.}


Proof Taking the Laplace transform with respect to $t$ to equations $(27) \sim(34)$, we have

$$
\begin{gathered}
\frac{\mathrm{d}}{\mathrm{d} x} \mathcal{Q}_{i}^{*}(s, x)+\left(s+r_{i+1} \lambda+\alpha(x)\right) \mathcal{Q}_{i}^{*}(s, x)=0,(i=0,1) \\
\frac{\mathrm{d}}{\mathrm{d} x} \mathcal{Q}_{2}^{*}(s, x)+\left(s+r_{2} \lambda+\alpha(x)\right) \mathcal{Q}_{2}^{*}(s, x)=r_{1} \lambda \mathcal{Q}_{0}^{*}(s, x) \\
\frac{\mathrm{d}}{\mathrm{d} x} \mathcal{Q}_{3}^{*}(s, x)+\left(s+r_{1} \lambda+\alpha(x)\right) \mathcal{Q}_{3}^{*}(s, x)=r_{2} \lambda \mathcal{Q}_{1}^{*}(s, x) \\
\left(s+r_{i} \lambda\right) \mathcal{Q}_{i+4}^{*}(s)=\int_{0}^{\infty} \mathcal{Q}_{i-1}^{*}(s, x) \alpha(x) \mathrm{d} x,(i=1,2) \\
\frac{\mathrm{d}}{\mathrm{d} y} \mathcal{Q}_{7}^{*}(s, y)+\left(s+r_{2} \lambda+\mu_{1}(y)\right) \mathcal{Q}_{7}^{*}(s, y)=0 \\
\frac{\mathrm{d}}{\mathrm{d} z} \mathcal{Q}_{8}^{*}(s, z)+\left(s+r_{1} \lambda+\mu_{2}(z)\right) \mathcal{Q}_{8}^{*}(s, z)=0 \\
\mathcal{Q}_{0}^{*}(s, 0)=\int_{0}^{\infty} \mathcal{Q}_{8}^{*}(s, z) \mu_{2}(z) \mathrm{d} z+1, \mathcal{Q}_{1}^{*}(s, 0)=\int_{0}^{\infty} \mathcal{Q}_{7}^{*}(s, y) \mu_{1}(y) \mathrm{d} y, \mathcal{Q}_{i}^{*}(s, 0)=0,(i=2,3) \\
\mathcal{Q}_{7}^{*}(s, 0)=\int_{0}^{\infty} \mathcal{Q}_{2}^{*}(s, x) \alpha(x) \mathrm{d} x+r_{1} \lambda \mathcal{Q}_{5}^{*}(s), \mathcal{Q}_{8}^{*}(s, 0)=\int_{0}^{\infty} \mathcal{Q}_{3}^{*}(s, x) \alpha(x) \mathrm{d} x+r_{2} \lambda \mathcal{Q}_{6}^{*}(s)
\end{gathered}
$$

According to the initial conditions, we have: $\mathcal{Q}_{5}^{*}(0)=\mathcal{Q}_{6}^{*}(0)=0$

The solutions can be written as

$$
\begin{gathered}
\mathcal{Q}_{i}^{*}(s, x)=\mathcal{Q}_{i}^{*}(s, 0) e^{-\left(s+r_{i+1} \lambda\right) x} V(x),(i=1,2), \quad \mathcal{Q}_{2}^{*}(s, x)=\frac{r_{1}}{r_{1}-r_{2}} \mathcal{Q}_{0}^{*}(s, 0) V(x)\left(e^{-\left(s+r_{2} \lambda\right) x}-e^{-\left(s+r_{1} \lambda\right) x}\right) \\
\mathcal{Q}_{3}^{*}(s, x)=\frac{r_{2}}{r_{1}-r_{2}} \mathcal{Q}_{1}^{*}(s, 0) V(x)\left(e^{-\left(s+r_{2} \lambda\right) x}-e^{-\left(s+r_{1} \lambda\right) x}\right), \quad \mathcal{Q}_{i+4}^{*}(s)=\frac{v^{*}\left(s+r_{i} \lambda\right)}{s+r_{i} \lambda} \mathcal{Q}_{i-1}^{*}(s, 0),(i=1,2) \\
\mathcal{Q}_{0}^{*}(s, 0)=\frac{\mathcal{Q}_{7}^{*}(s, y)=\mathcal{Q}_{7}^{*}(s, 0) e^{-\left(s+r_{2} \lambda\right) y} \bar{H}_{1}(y), \quad \mathcal{Q}_{8}^{*}(s, z)=\mathcal{Q}_{8}^{*}(s, 0) e^{-\left(s+r_{1} \lambda\right) z} \bar{H}_{2}(z)}{1-C_{1}(s) C_{2}(s) h_{1}^{*}\left(s+r_{2} \lambda\right) h_{2}^{*}\left(s+r_{1} \lambda\right)}, \quad \mathcal{Q}_{7}^{*}(s, 0)=\frac{C_{1}(s)}{1-C_{1}(s) C_{2}(s) h_{1}^{*}\left(s+r_{2} \lambda\right) h_{2}^{*}\left(s+r_{1} \lambda\right)} \\
\mathcal{Q}_{1}^{*}(s, 0)=\frac{C_{1}(s) h_{1}^{*}\left(s+r_{2} \lambda\right)}{1-C_{1}(s) C_{2}(s) h_{1}^{*}\left(s+r_{2} \lambda\right) h_{2}^{*}\left(s+r_{1} \lambda\right)}, \quad \mathcal{Q}_{8}^{*}(s, 0)=\frac{C_{1}(s) C_{2}(s) h_{1}^{*}\left(s+r_{2} \lambda\right)}{1-C_{1}(s) C_{2}(s) h_{1}^{*}\left(s+r_{2} \lambda\right) h_{2}^{*}\left(s+r_{1} \lambda\right)}
\end{gathered}
$$

The reliability of the system is

$$
R(t)=\sum_{i=0}^{3} \int_{0}^{\infty} \mathcal{Q}_{i}(t, x) \mathrm{d} x+\mathcal{Q}_{5}(t)+\mathcal{Q}_{6}(t)+\int_{0}^{\infty} \mathcal{Q}_{7}(t, y) \mathrm{d} y+\int_{0}^{\infty} \mathcal{Q}_{8}(t, z) \mathrm{d} z
$$

The Laplace transformation formula of the reliability of the system is 


$$
\begin{aligned}
R^{*}(s)= & \sum_{i=0}^{3} \int_{0}^{\infty} \mathcal{Q}_{i}^{*}(s, x) \mathrm{d} x+\mathcal{Q}_{5}^{*}(s)+\mathcal{Q}_{6}^{*}(s)+\int_{0}^{\infty} \mathcal{Q}_{7}^{*}(s, y) \mathrm{d} y+\int_{0}^{\infty} \mathcal{Q}_{8}^{*}(s, z) \mathrm{d} z \\
= & \frac{\left(r_{1} V^{*}\left(s+r_{2} \lambda\right)-r_{2} V^{*}\left(s+r_{1} \lambda\right)\right)\left(1+C_{1}(s) h_{1}^{*}\left(s+r_{2} \lambda\right)\right)}{\left(r_{1}-r_{2}\right)\left(1-C_{1}(s) C_{2}(s) h_{1}^{*}\left(s+r_{2} \lambda\right) h_{2}^{*}\left(s+r_{1} \lambda\right)\right)}+\frac{C_{1}(s) \bar{H}_{1}^{*}\left(s+r_{2} \lambda\right)+C_{1}(s) C_{2}(s) \bar{H}_{2}^{*}\left(s+r_{1} \lambda\right) h_{1}^{*}\left(s+r_{2} \lambda\right)}{1-C_{1}(s) C_{2}(s) h_{1}^{*}\left(s+r_{2} \lambda\right) h_{2}^{*}\left(s+r_{1} \lambda\right)} \\
& +\left(\frac{v^{*}\left(s+r_{1} \lambda\right)}{s+r_{1} \lambda}+\frac{C_{1}(s) h_{1}^{*}\left(s+r_{2} \lambda\right) v^{*}\left(s+r_{2} \lambda\right)}{s+r_{2} \lambda}\right) \frac{1}{1-C_{1}(s) C_{2}(s) h_{1}^{*}\left(s+r_{2} \lambda\right) h_{2}^{*}\left(s+r_{1} \lambda\right)}
\end{aligned}
$$

\section{References}

[1] R. E. Barlow, F. Proschan, Statistical theory of reliability and life testing: probability models, Holt, Rinehart and Winston, 1975.

[2] J. G. Shanthikumar, U. Sumita, General shock models associated with correlated renewal sequences., Journal of Applied Probability 20 (3) (1983) 600-614.

[3] J. G. Shanthikumar, U. Sumita, Distribution properties of the system failure time in a general shock model, Advances in Applied Probability 16 (1984) 363-377.

[4] Z. Li, P. Zhao, Reliability analysis on the -shock model of complex systems, IEEE Transactions on Reliability 56 (2) (2007) 340-348.

[5] K. Murari, V. Goyal, Comparison of two unit cold standby reliability models with three types of repair facilities, Microelectronics Reliability 24 (1) (1984) 35-49.

[6] R. Gupta, A. Chaudhary, A two-unit priority standby system subject to random shocks and releigh failure-time distribution, Microelectronics Reliability 32 (12) (1992) 1713-1723.

[7] R. Gupta, R. Kishan, On the profit comparison of two stochastic models each pertaining to a twounit standby system with fixed preparation time and hyper-exponential repair time distributions, International Journal of Systems Science 30 (12) (1999) 1309-1317.

[8] Y. Lam, A geometric process maintenance model with preventive repair, European Journal of Operational Research 182 (2) (2007) 806-819.

[9] Y. L. Zhang, G. J. Wang, A deteriorating cold standby repairable system with priority in use, European Journal of Operational Research 183 (1) (2007) 278-295. 
[10] M. A. W. Mahmoud, M. E. Moshref, On a two-unit cold standby system considering hardware, human error failures and preventive maintenance, Mathematical and Computer Modelling 51 (5-6) (2010) 736-745.

[11] B. T. Doshi, Queueing systems with vacations - a survey, Queueing Systems 1 (1) (1986) 29-66.

[12] J. Ke, K. Wang, Vacation policies for machine repair problem with two type spares, Applied Mathematical Modelling 31 (5) (2007) 880-894.

[13] B. Su, D. Shi, Reliability analysis of n-unit series systems with multiple vacations of a repairman, Mathematical Statistics and Applied Probability 10 (1) (1995) 78-82.

[14] J. Jia, S. Wu, A replacement policy for a repairable system with its repairman having multiple vacations, Computers and Industrial Engineering 57 (1) (2009) 156-160.

[15] J. Jia, S. Wu, Optimizing replacement policy for a cold-standby system with waiting repair times, Applied Mathematics and Computation 214 (1) (2009) 133-141.

[16] J. L. Chaudhry, J. G. C. Templeton, A First Course in Bulk Queue, Jon Wiley \& Sons Ltd, 1983.

[17] A. Birolini, Quality and Reliability of Technical Systems, Springer-Verlag, Berlin, 1994. 
[10] M. A. W. Mahmoud, M. E. Moshref, On a two-unit cold standby system considering hardware, human error failures and preventive maintenance, Mathematical and Computer Modelling 51 (5-6) (2010) 736-745.

[11] B. T. Doshi, Queueing systems with vacations - a survey, Queueing Systems 1 (1) (1986) 29-66.

[12] J. Ke, K. Wang, Vacation policies for machine repair problem with two type spares, Applied Mathematical Modelling 31 (5) (2007) 880-894.

[13] B. Su, D. Shi, Reliability analysis of n-unit series systems with multiple vacations of a repairman, Mathematical Statistics and Applied Probability 10 (1) (1995) 78-82.

[14] J. Jia, S. Wu, A replacement policy for a repairable system with its repairman having multiple vacations, Computers and Industrial Engineering 57 (1) (2009) 156-160.

[15] J. Jia, S. Wu, Optimizing replacement policy for a cold-standby system with waiting repair times, Applied Mathematics and Computation 214 (1) (2009) 133-141.

[16] J. L. Chaudhry, J. G. C. Templeton, A First Course in Bulk Queue, Jon Wiley \& Sons Ltd, 1983.

[17] A. Birolini, Quality and Reliability of Technical Systems, Springer-Verlag, Berlin, 1994. 